\title{
Germanica
}

\section{Die Neue Sachlichkeit}

Malerei der Weimarer Zeit

La peinture de la Nouvelle Objectivité

\section{Wieland Schmied}

\section{OpenEdition \\ Journals}

Édition électronique

URL : http://journals.openedition.org/germanica/2393

DOI : $10.4000 /$ germanica. 2393

ISSN : 2107-0784

Éditeur

Université de Lille

\section{Édition imprimée}

Date de publication : 31 décembre 1991

Pagination : $217-228$

ISSN : 0984-2632

Référence électronique

Wieland Schmied, «Die Neue Sachlichkeit », Germanica [Online], 9 | 1991, Online erschienen am: 25

Februar 2014, abgerufen am 06 Oktober 2020. URL : http://journals.openedition.org/germanica/2393 ; DOI : https://doi.org/10.4000/germanica.2393

Ce document a été généré automatiquement le 6 octobre 2020.

(C) Tous droits réservés 


\title{
Die Neue Sachlichkeit
}

\author{
Malerei der Weimarer Zeit \\ La peinture de la Nouvelle Objectivité
}

Wieland Schmied

1 Durch die deutsche Kunst des 20. Jahrhunderts zieht sich ein großer Dualismus. Er wird geprägt von expressionistischen und anti-expressionistischen Strömungen. Wir könnten mit dem Titel eines Bildes von Paul Klee auch von «Hauptwegen und Nebenwegen» sprechen. Der Hauptweg, das war zweifellos der Expressionismus. In seinem Zeichen beginnt die Moderne in Deutschland: 1905 mit der Künstlergemeinschaft «Brücke» in Dresden, rund fünf Jahre später mit der Gruppe um den «Blauen Reiter» in München.

2 Doch bald artikulieren sich Gegenbewegungen - unter dem Schock des Ersten Weltkrieges meldet sich Dada zu Wort, zuerst in Zürich, dann in Köln, Hannover, Berlin. Anfang der zwanziger Jahre beginnt man von einer neuen Malerei zu sprechen. Ihre unterschiedlichen Tendenzen werden als Verismus, als Neoklassizismus, als Neonaturalismus, als magischer Realismus, als neue Sachlichkeit benannt, auch die Bezeichnungen «Ingrismus» (nach dem französischen Klassizisten Ingres) und neue Romantik werden versuchsweise eingeführt. Schließlich setzt sich der Name «Neue Sachlichkeit» als Sammelbegriff durch.

3 So verschiedenartig die einzelnen Strömungen sind, die dieser Begriff vereint, in einem stimmen sie alle überein: in ihrer Absage an den Expressionismus. Und die Kritik und Museums weit bewertet sie, mag sie ihr teilweise auch noch so reserviert gegenüberstehen, als die Kunst, die den Expressionismus abgelöst, ja überwunden hat. Das kommt am deutlichsten in den beiden Manifestationen zum Ausdruck, die 1925 ihren ersten - und wohl schon entscheidenden - Höhepunkt markieren. Gustav F. Hartlaub, der frisch bestallte Direktor der Mannheimer Kunsthalle, gibt der von ihm initiierten Ausstellung den Titel: «Neue Sachlichkeit. Deutsche Malerei seit dem Expressionismus». Und Franz Roh, der Münchener Kritiker, nennt sein Buch (freilich darin auch einem Verlegerwunsch folgend) noch expliziter: «NachExpressionismus». 
Der Untertitel heißt dann: «Magischer Realismus. Probleme der neuesten europäischen Malerei».

4 Aber der Expressionismus, so oft totgesagt, war noch keineswegs erloschen. Zunächst koexistierte er im Weimarer Bauhaus fort (und lag mit den dort allmählich übermächtig werdenden konstruktivistischen Tendenzen in manchmal fruchtbarem, manchmal erbittertem Widerstreit). Nach dem Zweiten Weltkrieg lebte er zunächst im abstraktem Expressionismus diesseits und jenseits des Atlantiks wieder auf, erhob sein Haupt im Kreis der Cobra-Gruppe (und ihrer Ableger) und kulminierte im «Neoexpressionismus» der sogenannten «neuen Wilden» oder «neuen Heftigen».

5 Alle diese Bewegungen, die Malerei der «abstrakten Expressionisten» nicht weniger als die der der «neue Wilden» drei Jahrzehnte später, stießen oft gerade bei der sich selbst als progressiv empfindenden Kritik - die sich am Fortschritt der jeweils zuletzt etablierten Bewegung orientierte - zunächst auf Skepsis und Widerspruch. In besonderem Maße war das beim ersten Auftreten der Malerei der Neuen Sachlichkeit der Fall. Bedeutete ihr «Zurück zum Gegenstand» nicht in der Tat, kunstgeschichtlich gesehen, einen Rückschritt? So sah es jedenfalls Wilhelm Hausenstein, der im «erneuerten Interesse für die Welt... der äußeren Gegenständlichkeit» einen «Rückschlag» zu erkennen meinte. Selbst Hartlaub meldete in seinen ersten Reaktionen Zweifel an, die sich freilich besonders auf die aufreizend «häßlichen» Gegenstände bezogen, die sich einzelne Veristen - oder «Neonaturalisten» - gewählt hatten. Erst die eigene Ausstellung, ihr Ergebnis, ihr Echo, ihr Erfolg mögen ihn ganz von der Tragfähigkeit der neuen Richtung überzeugt haben.

6 Den kritisch-ablehnenden Stimmen - die auch Mitte der zwanziger Jahre noch keineswegs verstummt waren - nachdrücklich widersprechend schrieb Wilhelm Michel 1925 im 56. Band von «Deutsche Kunst und Dekoration» im Hinblick auf die Mannheimer Ausstellung: «Halten wir vor allem das eine fest: Es handelt sich um eine neue Sachlichkeit. Es handelt sich keineswegs um eine Rückkehr zur Sachlichkeit der vorexpressionistischen Zeit. Es handelt sich um die Ding-Entdeckung nach der Ich-Krise. Es handelt sich um eine -Ergreifung nach jener wichtigen Wandlung, die das schroff idealistische Zwischenspiel des letzten Jahrzehnts herbbeigeführt hat».

7 In dieser Kritik sind zwei wesentliche Charakteristika der Neuen Sachlichkeit angesprochen. Dabei ist das eine formaler, das andere Charakteristikum inhaltlicher Natur.

8 Zum einen: Die Neue Sachlichkeit stellt keine fraglose, sondern eine bewußt konstruierte Realität dar. Sie ist eine Form realistischer Kunst, entwickelt nach der Abstraktion, nach den ersten abstrakten Bildern eines Kandinsky, Kupka, Malewitsch, Mondrian, die unsere Anschauung der Welt und unser Verständnis der Kunst so nachhaltig erschüttert und verändert hatten. Sie ist eine Form realistischer Kunst, entwickelt nach den Erfahrungen Dadas und der heterogene Elemente verbindenden DadaCollage. Sie fügt die Fragmente einer zerbrochenen Wirklichkeit wieder behutsam collageartig zusammen, und man hat ihre Bilder darum auch zuweilen «handgemalte Collagen» genannt.

9 Zum zweiten. Die Neue Sachlichkeit versucht anders als Dada - das alles in Zweifel zog - und anders als der Expressionismus, der das eigene Ich, das innere Erleben gläubig und selbstüberzeugt auf die Folie der äußeren Wirklichkeit projezierte - sich wieder demütigvertrauensvoll (und mitunter auch verzweifelt) an der Realität der Außenwelt, der Welt der Dinge zu orientieren, auch wenn diese Dinge in der Kunst erst wieder neu 
aufgebaut, neu konstruiert werden mußten. Die Darstellung der Außenwelt war ihr wichtiger als jedes subjektive Bekenntnis. Das Ich mußte hinter den Objekten zurücktreten, mußte bescheiden werden.

10 Nach den Ekstasen des Expressionismus suchten die Künstler der Neuen Sachlichkeit die Nüchternheit des Blicks, nach den kosmischen Träumen die banalen Themen, nach dem Überschwang des Gefühls die Freiheit von aller Sentimentalität. Wo der Expressionismus nach den Sternen gegriffen hatte, wollte man wieder Boden unter den Füßen gewinnen. Nun, da die Apokalypse eingetreten war, war man des apokalyptischen Pathos müde. Durchlittene Schrecken, gegenwärtige Konflikte malt man anders als die Vision kommender Katastrophen. Nur in der Faszination durch den Rhythmus der modernen Metropolen, in der häufigen Fixierung an großstädtische Motive blieb man dem Expressionismus nahe.

11 Franz Roh hat in seinem Buch «Nach-Expressionismus. Magischer Realismus» die entscheidenden Merkmale expressionistischer und sachlicher Malerei in einer Übersicht einander gegenübergestellt:

\section{Expressionismus}

Ekstatische Gegenstände

Viel religiöse Vorwürfe

objekt unterdrückend

Rhythmisierend

Erregend

Ausschweifend

Dynamisch

Laut

Summarisch

Vordergründig (Nahbild)

Nach vorn treibend

Monumental

Warm

Dicke Farbsubstanz

Aufrauhend

Wie unbehauenes Gestein

Arbeitsprozeß (Faktur)

spüren lassend

Expressive Deformierung

der Objekte

Diagonalreich

(in Schrägen),

Gegen die Bildränder

Arbeitend

Urtümlich

13 Nach-Expressionismus

Nüchterne Gegenstände

Sehr wenig religiöse Vorwürfe

objekt verdeutlichend

Darstellend

Vertiefend 
eher streng, puristisch

Statisch

Still

Durchführend

Vorder- und hintergründig (Nahbild + Fernbild)

Auch zurückfliehend

Miniaturartig

Kühl, bis kalt

Dünne Farbschicht

Glättend, vertrieben

Wie blank gemachtes Metall

Arbeitsprozeß austilgend

(reine Objektivation)

Harmonische Reinigung

der Gegenstände

Eher rechtwinklig

dem Rahmen parallel

$\mathrm{Zu}$ ihnen festsitzend

Kultiviert

Die Kunst der Neuen Sachlichkeit war wie keine andere Ausdruck der Zeitstimmung der zwanziger Jahre. Ihre Bilder lesen sich wie ein Protokoll der Weimarer Zeit - ihrer Wünsche, Ideale, Enttäuschungen, ihrer Ausflüchte, Konflikte und Defizite. Im Refrain eines Schlagers hieß es damals: «Es liegt in der Luft eine Sachlichkeit». In kaltschnäuzig-schnippischem Ton werden in diesem Song die Fetische der neuen Zeit Luftschiffe, Flugzeuge, Automobil, Elektrizität, Radio, Schallplatte - besungen und zugleich die Werte einer vergangenen Epoche wie Liebe oder Empfindsamkeit verspottet. Sie erscheinen als Ballast, der bei der Bewältigung des Lebens in einer technisiserten Welt nur hinderlich ist:

Weg mit Schnörkel, Stuck und Schaden!

Glatt baut man die Hausfassaden!

Morgen baut man Häuser bloß,

ganz und gar fassadenlos...

Der Schlager spinnt diesen Gedanken genüßlichynisch bis zur letzten logischen Konsequenz aus:

Fort, die Möbel aus der Wohnung.

Fort mit was nicht hingehört.

Wir behaupten ohne Schonung:

jeder Mensch, der da ist, stört.

Die Maler der Neuen Sachlichkeit haben diese «in der Luft» liegende Stimmung gemalt, und sie haben sich zugleich, indem sie diese malten, indem sie sie in den luftleeren Raum ihrer Bilder übersetzten, gegen sie gewehrt. Sie waren sich bewußt, daß sie selbst zu den Menschen gehörten, die «störten». Damit versuchten sie fertig zu werden. Sie kannten den kaltschnäuzigzynischen Ton, der in der Zeit lag, sie waren ihm täglich ausgesetzt, nicht zuletzt in ihrer Kunst. Sie haben auf ihn reagiert, ohne

ihn ganz zu übernehmen. Ihre Empfindungen versteckten sie hinter den Dingen, und oft blieben sie unsichtbar. Dinge konnten nicht so zynisch sein wie Menschen - und waren auch nicht so verletzbar. Darum hielten sie sich an die Dinge, und ließen die Menschen nur zögernd in ihre Bilder treten. 
18 Nicht mehr verletzt werden - so ließe sich das erste Ziel der Künstler der Neuen Sachlichkeit benennen. Nicht mehr verletzt werden von der Sachlichkeit - und Herzlosigkeit - der Menschen und sich darum lieber gleich in den Schutz der Dinge begeben - so hätte ihre ungeschriebene Devise lauten können. Dann lieber gleich selber in den Angriff übergehen und die «Verletzer» demaskieren und attackieren - wie es George Grosz und Karl Hubbuch getan haben - oder die Wunden der Verletzten in erbarmungsloser, erschreckender Offenheit vorzeigen - wie Otto Dix und Rudolf Schlichter es taten.

19 Die Expressionisten malten gern den nackten Menschen - für Dix dagegen ist der nackte Mensch fast immer zugleich der verstümmelte Mensch, gezeichnet von Alter, Krankheit, Laster, Ausschweifung. Für Schad erscheint der nackte Körper der Frau als Objekt, als Sache, verfügbar, besitzbar, manipulierbar. Dix und Schad haben gerne tätowierte Haut gemalt: die Tätowierung ist für die Menschen da so etwas wie die verzweifelte Hoffnung, den zwangsläufigen Verfall des Körpers vielleicht ein wenig hinausschieben zu können.

Die Maler der Neuen Sachlichkeit stellen den Menschen lieber angezogen dar, in möglichst viele Hüllen gepackt. Sie malen ihn geschützt durch Anzüge, Westen, Krawatten, Lederjacken, Mäntel, durch Handschuhe, Hut oder Mütze. Bei Räder Scheidt steht der junge Mann in schwarzem Anzug mit gelben Handschuhen und Bowler allein auf dem weiten Platz vor den geometrischgenormten Architekturen, die direkt aus der Pittura metafisica Giorgio de Chiricos genommen und in die moderne Welt versetzt erscheinen. Er steckt in seinem eng ansitzenden Gewand wie in einem Panzer, geschützt vor Angst und Kälte, aber darum umso mehr isoliert von seiner Umgebung und umso einsamer. So hat auch Schad den heimatlosen Aristokraten Graf St. Génois durch seinen Smoking gewappnet, als wollte er ihm vor der Verführung durch die Sirenen des Salons bewahren. Und Carl Grossberg steckt seinen Protagonisten gar in eine Taucherausrüstung um ihn so für immer unverletzlich zu machen.

«Der Mensch ist gut», hatte der Expressionismus postuliert, «der Mensch ist ein Vieh», konterte George Grosz am Anfang der zwanziger Jahre. Bald aber wandelte sich dieses zynische Bekenntnis zur Einsicht: dem Menschen muß geholfen werden, er muß beschützt werden. Er ist vor dem kapitalistischen Ausbeuter und dem blutgierigen Militaristen geradeso zu beschützen, wie vor den bedrohlichen anonymen Mächten der Technik, der Großstadt, dem Fortschritt, der Welt der Maschine...

Die Maler der Neuen Sachlichkeit konzentrierten ihren Blick auf das Alltägliche, die eigene Umwelt, den eigenen Erfahrungsbereich - über den allein man Genaues auszusagen vermag -, den Asphalt der Großstadt oder den abseitigen Winkel. Das führte zur Scharfeinstellung der künstlerischen Optik auf wenig beachtete Details (wie in Ernst Thoms' «Kramladen») oder zur Entdeckung ungewohnter Perspektiven. In einem Bild von Ernst Thoms blickt man vom Dachboden - dem Ort der Außenseiter durch eine offene Luke tief hinunter in die Eingeweide des Hauses, bei Franz Radziwill schaut man gleichsam aus der Fischperspektive die nicht enden wollenden, schimmernden Flanken des Ozeanriesen hinauf. Die Wahl eines solchen Blickpunktes ist ebensowenig zufällig, wie die Wahl der Themen, sie drängt sich aus der Einstellung der Maler der Neuen Sachlichkeit geradezu auf: Es ist der Blick des kleinen Mannes, der sich den Phänomenen der modernen technischen Welt ausgesetzt sieht, die er nicht geschaffen hat, die er nicht beherrscht und die er nicht zu deuten vermag. Deswegen hält er sich auch so gern an die Dinge des täglichen Gebrauchs als Beispiele einer 
überschaubaren Welt. Übermächtig wird der kleine Mann auf dem Kai bei Radziwill von den gewaltigen Volumen der Überseeschiffe bedrängt, bis er wegblickt und sich in seine Zeitung versenkt. Hier wird ihm die Welt noch verständlich buchstabiert. In ihren vertrauten Klischees fühlt er sich zu Hause. Die Erfahrung der Technik dagegen bleibt ihm fremd und bedrohlich.

Für diese Bedrohung hat Carl Grossberg eindrucksvolle Metaphern gefunden. Affen und Fledermäuse bemächtigen sich zu abendlicher Stunde in unbewachten Augenblicken der verlassenen Maschinensäle, bedienen die technischen Apparate und Armaturen, drehen die Hebel und setzen die Kessel unter Druck. Der Schlaf der Maschinen gebiert Ungeheuer, könnte man Goya abwandeln. Nicht der Mensch beherrscht hier die Technik, er wird von ihr beherrscht. Die Einstellung zu ihren Erfindungen ist bei Grossberg und Davringhausen, bei Radziwill und Georg Scholz nicht die des Ingenieurs und Konstrukteurs, der sie erdenkt, entwirft und baut, sondern die des Opfers, das ihnen preisgegeben ist, dem diese Konstruktionen fremd und rätselhaft bleiben. Von allen technischen Erfindungen werden wohl nur Glühlampe und Telefon fraglos akzeptiert, finden nur Grammophon und Schallplatte ungeteilten Beifall.

Die Maler der Neuen Sachlichkeit entstammen alle einer Generation. Sie kamen um 1890 oder in den neunziger Jahren des vorigen Jahrunderts zur Welt. So wurden geboren:

1894 H.M. Davringhausen, Carl Grossberg, Otto Nagel, Christian Schad, F.W. Seiwert, Gert H. Wollheim

1895 Otto Griebel, Heinrich Hoerle, Franz Radziwill

1896 Carl Barth, Nikiaus Stöcklin, Ernst Thoms.

Am Ende des Ersten Weltkriegs waren sie alle noch keine dreißig Jahre alt. Fast alle haben sie die Schrecken des Weltkriegs am eigenen Leib erlebt (oder waren vor ihnen, wie Schad, in die Schweiz geflüchtet); fast alle haben sie eine expressionistische, futuristische, dadaistische Epoche durchlaufen: umso abrupter und umso radikaler war dann bei den meisten von ihnen die Abwendung von Expressionismus und Dada, nur bei Dix blieb der kompositorische Furor des einstigen Expressionisten immer spürbar, er konnte seine Gestalten mit unbezwingbar expressiver Kraft zueinander ins Bild zwingen, mußte sie nicht mühsam wie die anderen nach Art der Collage zusammenfügen.

Standen für die Maler der Neuen Sachlichkeit Formprobleme auch nie im Vordergrund, so haben sie alle doch bewußt sich jene Mittel zurechtzulegen verstanden, die ihnen zur Erfassung und möglichst direkten, unverstellten Wiedergabe der Realität die geeignetsten erschienen. Sie alle glaubten nicht an Visionen. Große Visionen hatten die Menschen ins Unglück gestürzt, und dieses Unglück war von großen Begriffen und großen Worten begleitet gewesen. Dem mißtrauten sie. Dem galt ihre Absage. 

akademische Ausdrucksformen am Ende des Jahrzehnts ? Ist es die allgemein um sich greifende Unsicherheit, die alles Gewonnene wieder zu gefährden droht? Ist es eine weltweit sich rührende Tendenz zum einfachen Leben, zur heilen Welt, zur Geborgenheit in der Provinzialität, die auch unsere Maler ergreift ? Sind es die Schatten kommender Katastrophen, die sie resignieren und sich nach einem schützenden Unterschlupf umsehen lassen ? Oder ist es das komplexe Zusammenwirken einer Vielzahl von Ursachen?

Die Künstler der «Neuen Sachlichkeit» haben keine neuen Visionen hervorgebracht. Das unterscheidet sie von den Expressionisten wie von den Künstlern des Bauhauses. Auch ihr politisches Engagement galt weniger neuen Visionen oder Utopien, als vielmehr konkreten und greifbaren Zielen, war eher von Mitleid mit den Entrechteten oder Wut über den Zynismus der Herrschenden geprägt. Und es war nicht von Dauer.

Das drückt sich in den einzelnen Künstlerviten wie in der Entwicklung aus, die die Neue Sachlichkeit insgesamt genommen hat. Finden wir die ihr zugehörigen Maler zu Anfang der zwanziger Jahre alle in einer mehr oder weniger revolutionären Phase, von gärender Unruhe erfüllt, im Formalen nach den ihnen entsprechenden Mitteln suchend, politisch zu radikalen Standpunkten neigend, oft leidenschaftlich engagiert, von den schmerzhaften Erfahrungen des Krieges so wenig gelöst, wie vom Traum der Revolution, so haben sie Mitte der zwanziger Jahre ihre Form gefunden und sind dabei, ihrer Epoche in einer Reihe von zu äußerster Schärfe geschliffenen Bildern den adäquaten Ausdruck zu geben.

Um 1928/29 beginnt sich allmählich ein Verfall der Kraft, eine Aufweichung der Formen zugunsten eines unverbindlichen Post- Impressionismus abzuzeichnen. Die Spannung schwindet, der innere Antrieb erlahmt. Politische Zielsetzungen haben sich als unerfüllbar erwiesen, Hoffnungen sind zerbrochen. Viele Künstler suchen, desillusioniert und irritiert, in diesen Jahren Unterschlupf bei der Kirche. So sind damals kurz nacheinander Schlichter und Scholz - vorher eher in der «Roten Gruppe» zu Hause - zum katholischen Glauben konvertiert. sozialen Entwicklungen der Zeit suchen. Sie liegen zumindest ebenso in der persönlichen Biographie der einzelnen Künstler beschlossen.

dreißig. Die Nachkriegs jähre brachten für sie zugleich die aufgeschobenen Konflikte zwischen Pubertät und Geborgenheit in der Familie, zwischen Bohème und Bürgertum, anarchischer Gefühlswelt und überkommener Ordnung. Diese Konflikte multiplizierten sich durch die Zeitumstände, ihr Wertechaos, ihre Umstürze, die Träume von einer neuen Republik...

Mitte der zwanziger Jahre hat sich die Lebenssituation der Künstler in vielen Fällen entscheidend geändert. Sie haben ihren - wenn auch bescheidenen - Platz in der Gesellschaft gefunden. Beruflich beginnen sich erste Erfolge einzustellen, Bildverkäufe versprechen eine schmale, aber kontinuierliche Existenzgrundlage, einige sind als Lehrer an Akademien verpflichtet, viele im sicheren Hafen der Ehe gelandet, und es drängt sie, Familienglück und Kindersegen zum Thema ihrer Bilder zu machen.

Doch wo liegen die Gründe für die neuerliche Wende, den Rückfall in problemlosAls die Nationalsozialisten 1933 die Macht übernehmen, ist die Kraft der Maler der Neuen Sachlichkeit längst gebrochen. Jetzt sehen sie sich von Ausstellungs- und 
Malverboten verfolgt, müssen - soweit sie je solche erlangt hatten - Professuren aufgeben, viele Bilder von ihnen werden als entartet beschlagnahmt. Ihr Schicksal ist das der «inneren Emigration». Einige von ihnen - wie Schad - hören fast ganz zu malen auf, andere - wie Dix - haben in dieser Zeit des Rückzugs und des SichVerbergens noch bedeutende Bilder geschaffen, allegorische Sinnbilder der Zeit oder landschaftliche Panoramen aus dem Atem der Donauschule. Und für jeden, der diese im Verborgenen entstandenen Bilder - damals oder später - gesehen hat, wurde klar, daß dieses «Gespräch über Bäume» zugleich das beredte Schweigen über das Unheil umfaßte, das sich in den Tagen des «Dritten Reiches» vollzog.

Nach dem Krieg hat es lange gedauert, bis die Malerei der Neuen Sachlichkeit wiederentdeckt und in ihrem wahren Rang erkannt wurde. Erst um 1960, als aus England und den USA die ersten Bilder der Pop-Art herüberkamen, wagte man es, sich der eigenen neuen Gegenständlichkeit zu erinnern, die in den zwanziger Jahren ein europäischer Faktor gewesen war.

Als Stilrichtung ist die Neue Sachlichkeit unter den Künstlern der Nachkriegszeit nicht wieder aufgelebt. Zuviel war geschehen, als daß man an ihrer Bildwelt hätte unmittelbar anknüpfen können. Allerdings kam es zu einzelnen Gruppierungen, die sich mehr oder weniger auf die Maler der Neuen Sachlichkeit - oder des Magischen Realismus - berufen haben, so die norddeutsche Zebra-Gruppe (um Asmus, Nagel, Ullrich) oder die Berliner «Schule der neuen Prächtigkeit» (um Grützke, Bluth und Koeppel). Auch wichtige Einzelgänger, wie der Hamburger Harald Duwe oder der Nürnberger Michael Prechtel, haben ihre Inspiration durch den Realismus der zwanziger Jahre nie verleugnet.

Am eindeutigsten haben sich die Berliner kritisch engagierten Realisten - u.a. Baehr, Diehl, Petrick, Sorge, Vogelgesang - zu den Malern der Weimarer Zeit bekannt. In den Mittelpunkt einer großen Wanderausstellung, die in den siebziger Jahren durch viele europäische Länder unterwegs war, stellten sie - als Hommage an Otto Dix - eine im Maßstab 1:1 ausgeführte Reproduktion seines Großstadt-Triptychons. Doch ihre Abhängigkeit von den Vorbildern der Neuen Sachlichkeit ist alles in allem gewiß nicht größer, als die der «Neuen Wilden» von den Künstlern der «Brücke».

Mag die «Neue Sachlichkeit» insgesamt auch nicht mehr als ein Nebenweg der deutschen Kunst des 20. Jahrhunderts gewesen sein, so war sie doch ein höchst bedeutsamer. Bedeutsam nicht nur als Dokument der Zeit, das uns Geist und Ungeist der Epoche getreulich überliefert, sondern bedeutsam auch als malerische Manifestation von hohem Rang.

\section{RÉSUMÉS}

Die Neue Sachlichkeit ist ein Sammelbegriff für unterschiedliche Richtungen, deren gemeinsamer Nenner die Ablehnung, ja Überwindung des Expressionismus ist. Das Motto «Zurück zum Gegenstand» ist nicht als Rückschritt nach den ersten Vorstößen der Abstraktion $\mathrm{zu}$ deuten, sondern vielmehr als «Dingentdeckung nach der Ich-Krise». Die bewußte 
Konstruktion der Realität, in der Form von «handgemalten Collagen» (Erinnerung an die DadaExperimente) ist ein Versuch, sich an der Welt der Dinge zu orientieren, unter Verzicht auf das subjektive Bekenntnis und auf das expressionistische Pathos. Die Menschen treten nur zögernd in die Bilder, gehüllt, schutzbedürftig. Die dominante Perspektive ist die des kleinen Mannes, der sich der modernen technischen Welt ausgesetzt sieht, die er nicht geschaffen hat. Diese Generation, die die Schrecken des Krieges am eigenen Leibe erlebt hat, schreckt vor hochgegriffenen Visionen zurück. Der Höhepunkt ihrer künstlerischen Leistung erreicht sie Mitte der Zwanziger Jahre, schon um 1928/1929 hat sie mit der allgemeinen Desillusionierung ihre Formkraft eingebüßt. In der Nazi-Zeit flüchten sich die meisten in die innere Emigration. Die Neue Sachlichkeit wird im Zuge der Pop-Art aus England und den USA in den sechziger Jahren wieder entdeckt als bedeutsame malerische Manifestation der deutschen Kunst des 20. Jahrhunderts.

La « Nouvelle Objectivité » est un terme général recouvrant des orientations artistiques diverses, qui ont toutes en commun le refus, voire le dépassement de l'expressionnisme. Le mot d'ordre du «retour à l'objet» n'est pas à prendre comme une régression après les premières avancées de l'abstraction, mais bien plutôt comme "découverte de la chose, après la crise du moi ». En construisant sciemment leur réalité sous forme de "collages peints à la main » (souvenir des expériences dada), les peintres de la Neue Sachlichkeit tentent de s'orienter dans le monde des choses, en renonçant tant à la confession subjective qu'au pathos expressionniste. Les hommes n'apparaissent qu'avec discrétion sur ces tableaux, comme des êtres fragiles, protégés par leurs vêtements. La perspective dominante est celle de l'homme dans sa petitesse, livré sans défense au monde moderne de la technique dont il n'est pas le créateur. Cette génération, qui a vécu les horreurs de la guerre, se défend des visions grandioses. Le sommet de sa production artistique se situe au milieu des années 20, les années 1928/1929, marquent le déclin de sa force créatrice, sous le poids de la désillusion générale. A l'époque nazie, la plupart de ces artistes se réfugient dans l'émigration intérieure. C'est dans les années 60, avec la vague du Pop-Art venue d'Angleterre et des Etats-Unis, que l'on redécouvre la Nouvelle Objectivité comme un courant important de la peinture allemande du $\mathrm{XX}^{\mathrm{e}}$ siècle. 